\title{
One-dimensional turbulent mass transfer at air-water interfaces: details of discontinuities of derivatives using the RSW method
}

\author{
B. B. Gonçalves ${ }^{1}$ \& H. E. Schulz $z^{1,2}$ \\ ${ }^{1}$ Department of Hydraulics and Sanitary Engineering, \\ University of São Paulo, Brazil \\ ${ }^{2}$ Nucleus of Thermal Sciences and Engineering, \\ University of São Paulo, Brazil
}

\begin{abstract}
The one-dimensional turbulent mass transfer was quantified using the nonlinear unclosed statistical governing equations derived from the traditional statistical methods. Further, this study considers the a priori simplifications of the bimodal Random Square Waves (RSW) approximation. This model enables the formulation of parametric equations for the variables of the statistical governing equations, but intrinsic aspects of this method still need to be clarified. In this sense, this study considers details of a version of the RSW equation for turbulent mass transfer which uses a constant reduction function. The mentioned details are related to discontinuities of higher order derivatives of the nondimensional concentration profile in the vicinity of a singular point. Numerical integrations were conducted at the air-water interface in both directions: from the liquid to the gas phase, and from the gas to the liquid phase. The results suggest that the continuity and smoothness of the calculated concentration profile does not depend on the discontinuities of the higher order derivatives, but the behaviour of the higher order derivatives depends strongly on the values of the nondimensional parameters existing in the formulation. Adequate boundary conditions were defined in the liquid phase, involving a new condition for the second derivative of the concentration profile. A fourth order Runge-Kutta method was used. This paper presents the proposed methodology; the comparison between results of calculated and measured concentration profiles,
\end{abstract}


the evolution of the profiles of the first, second and third space derivatives, and discussions concerning the adequacy of the results.

Keywords: turbulent flows, interfacial mass transfer, RSW method.

\section{Introduction}

The quantification of turbulent interfacial mass transport is of practical interest in industrial processes and in environmental problems, like the absorption of greenhouse gases by the oceans, or the absorption of oxygen by rivers. Davidson [1] mentions that turbulence is better quantified through statistical methods, because there are still no alternative methods that allow quantifying turbulence for general practical applications. But even these methods are mathematically complex, because nonlinear unclosed statistical governing equations are generated. Thus, turbulence is still not definitively quantified, so that ad hoc models are used together with numerical codes to obtain predictions of turbulent flows and related transport phenomena. Among the possibilities of quantifying the turbulent mass transfer, the use of a priori simplifications on bi-modal Random Square Waves (RSW) proved to be promising. The method generates parametric equations for the variables of the statistical governing equations.

Schulz et al. [2, 3] made a distinction between a priori and a posteriori models, the first involving ideal turbulence records (Random Square Waves) and a finite number of correcting parameters; and the second involving ad hoc models for the products of fluctuations of the dependent variables. An a priori model sets the correcting parameters prior to the statistical calculations, so that all statistical products involve only these parameters. An a posteriori model defines new equations or physical parameters for the new fluctuation products that arise after applying the statistical procedures (sometimes called ad hoc or heuristic approximations).

The present study uses a bi-modal Random Square Waves approximation (RSW), already used by Janzen [4] Schulz et al. [2, 3] and Schulz and Janzen [5]. It is generally accepted, in interfacial mass transfer studies, that the molecular diffusivity is the most relevant transport mechanism close to the surface, while the turbulent transport becomes relevant below this region (Komori et al. [6]). When considering low soluble gases, the gas phase imposes the boundary conditions at the interface (saturation concentration, for example), while all transport calculations can be done for the liquid phase, involving both molecular and turbulent mechanisms (Schulz and Janzen [5], Herlina and Jirka [7], Janzen et al. [8,9], for example). This is the situation considered in the present study.

Lopes Júnior [10] and Lopes Júnior and Schulz [11] analyzed the sensitivity of calculated profiles to variations in the boundary conditions. These studies considered the equation obtained for a constant reduction function, showing that some sets of parameters generate good solutions, while small modifications in the parameters could alter the results. The RWS equation obtained for a constant reduction function was then subjected to a more detailed study, concentrated around a singular point in the domain of calculus. The present paper shows 
results of numerical integrations for the nondimensional concentration profile conducted in both directions: from the liquid to the gas phase, and from the gas to the liquid phase, using adequate boundary conditions. This approach allowed observing the features of the higher order derivatives of the concentration profile in the vicinity of the singular point.

\subsection{Governing equations}

The general one dimensional mass conservation equation, without sources and sinks is given by:

$$
\frac{\partial \bar{C}}{\partial t}=\frac{\partial}{\partial z}\left(D \frac{\partial \bar{C}}{\partial z}-\overline{v c}\right)
$$

$\bar{C}$ and $c$ are the mean concentration and the concentration fluctuation, respectively, $v$ is the vertical velocity fluctuation, $t$ is the time, $z$ is the vertical coordinate and $D$ is the diffusion coefficient. Eqn (1) involves two unknown variables, $\bar{C}$ and $\overline{v c}$, so that "to close" this scalar transport problem needs an additional equation. An equation for the transport of $\overline{v c}$ could be constructed using the instantaneous conservation equations, already knowing that new statistical products appear. In the present approximation, the general equation for central moments was used, as follows:

$$
\frac{1}{\theta} \frac{\partial \overline{c^{\theta}}}{\partial t}+\overline{c^{\theta-1}} \frac{\partial \bar{C}}{\partial t}+\overline{v c^{\theta-1}} \frac{\partial \bar{C}}{\partial z}+\frac{1}{\theta} \frac{\partial \overline{v c^{\theta}}}{\partial z}=D\left(\overline{c^{\theta}-1} \frac{\partial^{2} \bar{C}}{\partial z^{2}}+\overline{c^{\theta-1} \frac{\partial^{2} c}{\partial z^{2}}}\right)
$$

Eqn (2) also reproduces eqn (1) for $\theta=1$ (that is, the first order central moment). The second equation is obtained for $\theta=2$ (the second order central moment), which involves $\overline{v c}$, but also a number of new statistical parameters, showing that new equations are always needed (higher order moments). The RSW method is used here to obtain a closed set of equations.

\section{Methodology - random square waves}

The methodology is described in Schulz et al. [2, 3] and Schulz and Janzen [5]. The authors considered mass concentration fluctuations between the interface and the bulk liquid like those sketched in fig. 1, dependent on $z$ and $t$. Fluctuations occur with values in the range between the upper and lower concentrations, $C_{p}$ and $C_{n}$, respectively (fig. 2(a)). The methodology assumes that the turbulent records may be simplified through a random square wave, as schematized in fig. 2(b). The ideal oscillations vary between the extreme values, following a bimodal character. But real oscillations do not assume always this maximum amplitude, being necessary to impose a reduction, firstly done through the variables $P$ and $N$ shown in fig. 2(b), and further represented by a reduction function $\alpha$. Additionally, concentration oscillations depend on the velocity oscillations, but it does not imply that both fluctuations are superposed in time. 
So, a superposition coefficient, $\beta$, was also defined. Finally, the time fractions in which a point in space $(z)$ is subjected to the upper or the lower concentrations were considered. It was shown that the time fraction for the upper concentration, denoted by $n$, which varies along $z$, corresponds to the normalized concentration profile. It was named "partition function", because of the time partition between $C_{p}$ and $C_{n}$.

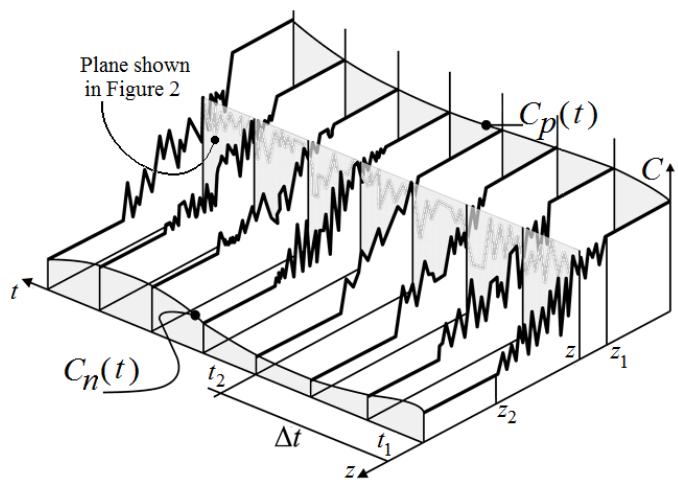

Figure 1: Random concentration field $C(z, t)$ oscillating between the boundary functions $C_{p}(t)$ and $C_{n}(t)$. Adapted from Schulz et al. [2].

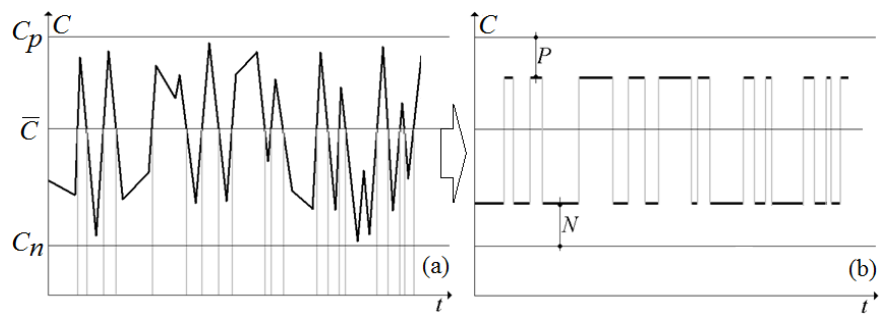

Figure 2: $\quad$ Sketch of a $C$ record at a position z. (a) as shown in fig. 1, (b) simplified to a bimodal random square wave. Adapted from Schulz et al. [3].

\subsection{RSW functions}

The partition function $n$, the reduction function $\alpha$, the superposition function $\beta$, and the usual rms velocity may vary between the values presented in table 1 . Eqns (1) and (2) were then transformed, through adequate operations involving $n, \alpha, \beta$ and $\sqrt{\overline{v^{2}}}$ (Schulz et al. [2,3]), into a new set of equations for these functions. As can be inferred, four equations would be needed to have a closed set of equations. Because the equations are nonlinear, the four coupled equations may have no simple solution. The referred studies have shown that the basic functions $\beta$ and $\sqrt{\overline{v^{2}}}$, appear always together, allowing writing a combined 
Table 1: Characteristics of the functions defined for one-dimensional scalar transport. Adapted from Schulz et al. [3].

\begin{tabular}{|c|c|c|c|c|}
\hline Function & $n$ & $\alpha$ & $\beta$ & $\sqrt{\overline{v^{2}}}$ \\
\hline Dimension & No & No & No & Velocity \\
\hline Physical ground & Partition & Reduction & Superposition & Reference velocity \\
\hline Maximum value & 1 & 1 & 1 & Undetermined \\
\hline Minimum value & 0 & 0 & 0 & 0 \\
\hline
\end{tabular}

function for them and reducing the previous system to a set of three equations. Further, the reduction function $\alpha$ was assumed as a constant (the version of the problem studied here), which reduced the system to two coupled equations. Finally, through simple substitution a single equation for $n$ was obtained, as:

$$
\begin{aligned}
& A\left[2 A n(1-n)+\frac{(1-2 n)}{2}\right] \frac{\mathrm{d}^{3} n}{\mathrm{~d} z^{*}} \frac{\mathrm{d} n}{\mathrm{~d} z^{*}}+ \\
& +A\left\{\begin{array}{l}
\left.-\left[2 A n(1-n)+\frac{(1-2 n)}{2}\right] \frac{\mathrm{d}^{2} n}{\mathrm{~d} z^{*^{2}}}+\kappa(1-n)\left[\frac{2 n(A-1)+1}{2}\right]+\right] \\
+\frac{\{1+2 A[A(1-2 n)-1]\}}{A}\left(\frac{\mathrm{d} n}{\mathrm{~d} z^{*}}\right)^{2}
\end{array}\right\} \frac{\mathrm{d}^{2} n}{\mathrm{~d} z^{* 2}}+ \\
& +\kappa\left\{(A-1)(1-n)-A\left[A(1-2 n)-\left(\frac{3}{2}-2 n\right)\right]\right\}\left(\frac{\mathrm{d} n}{\mathrm{~d} z^{*}}\right)^{2}=0 .
\end{aligned}
$$

$A=(1-\alpha), k=K\left(z_{2}-z_{1}\right)^{2} / D$ and $z^{*}=z /\left(z_{2}-z_{1}\right)$ are nondimensional parameters (see fig. 1 for $Z_{2}-Z_{1}$ ). $K$ is the interfacial mass transfer coefficient. Eqn (3) was solved here using the fourth order Runge-Kutta method, using convenient values of $A$ and $k$.

\section{Numerical solution}

\subsection{Some aspects of the "constant $\alpha$ " formulation}

In order to obtain a solution, adequate boundary conditions must be used. In practical applications the thickness of the boundary layer is defined as the position where the nondimensional concentration is $1 \%$ (that is, $99 \%$ of the concentration variation occurs inside the boundary layer). But no information of quantities like derivatives is furnished by this definition. Schulz et al. [2, 3], Lopes Júnior [10] and Lopes Júnior and Schulz [11] showed that different values of $A, k$, and derivatives of $n$ as boundary conditions apparently furnish viable solutions for $n$, so that some criteria would be needed to restrict the multiple possibilities. The practical definition of the boundary layer was not used in the present calculations. Only when comparisons were performed, the definitions were unified. Instead, the following boundary conditions were considered here: 
$n\left(z^{*}=0\right)=n(0)=1, n(1)=0, n^{\prime}(1)=0$. Using these values, eqn (3) allows obtaining a new boundary condition at $z^{*}=1: n$ " $(1)=0$ or $n$ " $(1)=k$. The positive value $n$ " $(1)=k$ assures a global minimum for $n$ at $z^{*}=1$, which is physically expected.

Janzen [4] obtained experimental results for $k$ in the range from 0.003 to 0.004 , used as reference for the present study. The system of equations derived from eqn (3) for use in explicit methods is given by:

$\frac{\mathrm{d} n}{\mathrm{~d} z^{*}}=j, \frac{\mathrm{d} j}{\mathrm{~d} z^{*}}=w, \quad \frac{\mathrm{d} w}{\mathrm{~d} z^{*}}=\frac{f_{1}+f_{2}}{f_{3}}$

where

$$
\left.\begin{array}{l}
f_{1}=-A\left\{\begin{array}{l}
-\left[2 A n(1-n)+\frac{(1-2 n)}{2}\right] w+\kappa(1-n)\left[\frac{2 n(A-1)+1}{2}\right]+ \\
+\frac{\{1+2 A[A(1-2 n)-1]\}}{A} j^{2}
\end{array}\right\} w, \\
f_{2}=-\kappa\left\{(A-1)(1-n)-A\left[A(1-2 n)-\left(\frac{3}{2}-2 n\right)\right]\right\} j^{2},
\end{array}\right\}
$$

and

$$
f_{3}=A\left[2 A n(1-n)+\frac{(1-2 n)}{2}\right] j \text {. }
$$

The function $f_{3}$ appears in the denominator of $\mathrm{d} w / \mathrm{dz} z^{*}=\left(f_{1}+f_{2}\right) / f_{3}$, and annuls at

$$
n=\frac{2 A-1+\sqrt{4 A^{2}+1}}{4 A} \quad \text { or } \quad A=\frac{2 n-1}{4 n(1-n)} .
$$

Because $A$ varies in the range [0,1], $f_{3}$ annuls for $n$ in the range [0.5, 0.809]. A theoretical solution exists for $n$ using $A=0$, not subjected to the abovementioned restriction (Schulz et al. [2, 3], based on eqn 3). From eqn 3 it is possible to suggest an internal boundary condition at the position defined by eqn (5), as:

$$
\begin{aligned}
& \left.A\left\{\kappa\left(1-n_{d}\right)\left[\frac{2 n_{d}(A-1)+1}{2}\right]+\frac{\left\{1+2 A\left[A\left(1-2 n_{d}\right)-1\right]\right\}}{A}\left(\frac{\mathrm{d} n}{\mathrm{~d} z^{*}}\right)_{d}^{2}\right\} \frac{\mathrm{d}^{2} n}{\mathrm{~d} z^{*}{ }^{2}}\right|_{d}+ \\
& +\kappa\left\{(A-1)\left(1-n_{d}\right)-A\left[A\left(1-2 n_{d}\right)-\left(\frac{3}{2}-2 n_{d}\right)\right]\right\}\left(\frac{\mathrm{d} n}{\mathrm{~d} z^{*}}\right)_{d}^{2}=0 .
\end{aligned}
$$

$n_{d}$ is the value of $n$ that satisfies eqn (5), and the subscript $d$ indicates that the conditions are taken at the discontinuity (at the singular point). Eqn (6) assumes that the first parcel of eqn (5) is zero. However, in order to verify if realistic solutions may be expected for $n$, previous studies applied the Runge Kutta method to eqns (4) using all boundary conditions at $z^{*}=0$ and $z^{*}=1$. As mentioned, different sets of parameters seemed to produce viable solutions. 
Having then obtained first solutions, the priority turned to verify how the internal aspects of the formulation allowed obtaining these solutions. Thus, the aim in applying explicit methods in the present study was to observe the behavior of the function $n$ and its derivatives close to the point of discontinuity (singular point), verifying if the integration is possible, that is, if the integrals of higher order derivatives (second and third orders) may be viewed as cases of convergent improper integrals.

\subsection{Applying the method}

The Runge-Kutta method needs a value of the third derivative at the origin, in order to start the calculations. The origin was first chosen as the point $\left(n, z^{*}\right)=$ $(0,1)$, with the calculations advancing to $(1,0)$. The parameters $k$ and $A$ must be imposed. In this study, the values of $k$ were 0.0029 and 0.003 (based on Janzen [4]). The value of $n$ for the discontinuity (singular point) was maintained close to 0.7 (in the range from 0.675 to 0.730 ). From eqn (5), $A$ varied in the range from $\sim 0.398$ to $\sim 0.583$. It was observed that, for the adopted $k$ values, this range allowed obtaining solutions converging to $n=1$ at $z^{*}=0$. The third derivative at $z^{*=1}$ was chosen by trial and error, in order to attain $n=1 \pm 0.1 \%$ at $z^{*=}=0$. In this sense, the third derivative was adjusted to fill the fixed boundary condition $n=1$ at $z^{*}=0$, and assumed values in the range of -0.04 to -0.03 . It was thus necessary to "cross" the singular point (discontinuities of the higher order derivatives) during the calculations, but the obtained solutions for $n$ were smooth and continuous. Fig. 3 shows the behavior of $n, n$ ', $n$ " and $n$ "', for $k=0.003$, $A \approx 0.3982$, and $n^{\prime \prime \prime}(1) \approx-0.0370$. The iteration step was $\Delta z^{*}=1 \times 10^{-3}$, the discontinuities occurred at $z_{d} * \approx 0.0784$, and $n(0) \approx 1.0008$.

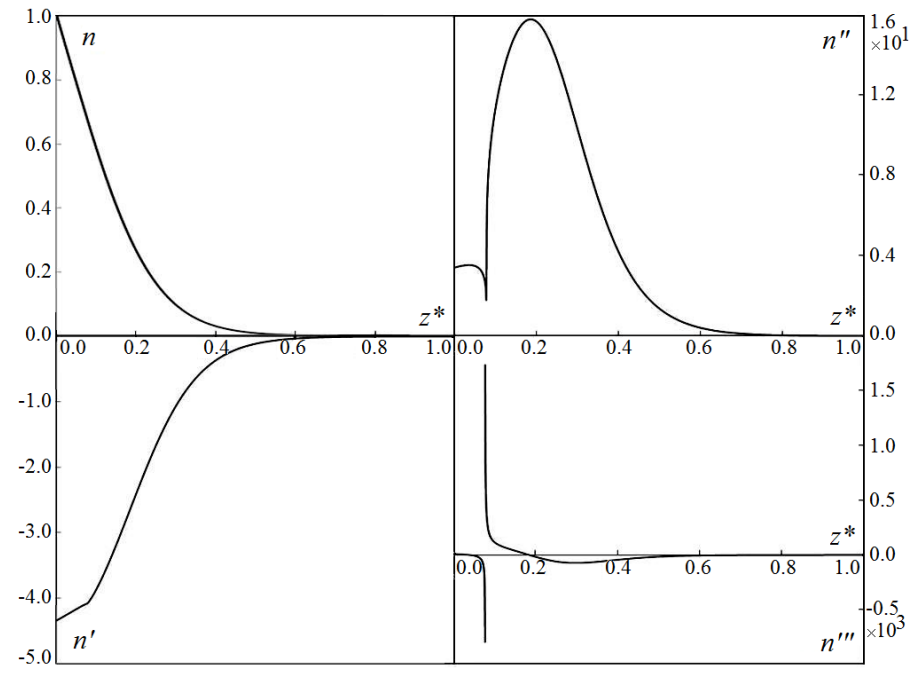

Figure 3: Smooth behavior of $n$, despite the discontinuities observed for higher order derivatives. $k=0.003, A \approx 0.3982$ and $n$ "' $(1) \approx-0.0370$. 
As can be seen, $n$ "' tends to $\pm \infty$ around $z_{d}{ }^{*}$. It do affects strongly $n$ ”, but it is not possible to infer that $n$ " tends to $-\infty$ at this position. Eqn (3) shows that the first parcel is undetermined at $z_{d} *$ (product of zero and $\pm \infty$, ideally being zero), so that a finite value for $n$ " would be possible. In the sequence of the graphs, the first derivative appears as continuous, although the graph is not necessarily smooth (different slopes at both sides of $z_{d}{ }^{*}$ ). The interfacial region is viewed as composed by sub-regions subjected to different transport mechanisms (see Herlina and Jirka [7] and Schulz and Janzen [5], for example); and different behaviors of the profiles, if representative of the phenomenon, should be correlated with these sub-regions. Despite the characteristics of the derivatives, the function $n$ is smooth and continuous, following observed behaviors.

\subsection{Comparison with measured data}

The prediction of $n$ shown in fig. 3 was compared with experimental data of Janzen [4]. In order to adopt the same vertical dimension for both sets of data, the usual definition of boundary layers was applied, that is, the nondimensional boundary layer thickness, $\delta=1$, is attained at $n=0.01$. Fig. 4 shows that the calculated and measured $n$ values present similar behaviors.

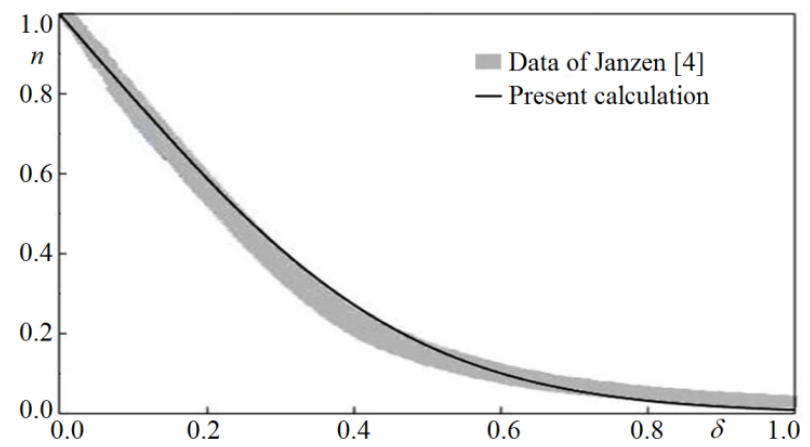

Figure 4: $\quad$ Measured and calculated $n$. The practical definition of the boundary layer was used in nondimensional form $(n=0.01$ at $\delta=1.0)$.

\subsection{Refined calculations close to the singular point}

As mentioned, different values of $A$ and $k$ were tested. It was observed that different behaviors of the derivatives could be obtained. However, in all these cases, the $n$ profiles showed smooth and continue characteristics. The mentioned behaviors are shown in figs. 5(a) and 5(b).

The differences observed between the graphs of figs. 5(a) and 5(b), mainly for the second and third derivatives, are a consequence of the different values of $k$ and $A$ adopted in the calculations. These parameters strongly affect the two derivatives, but do not affect the expected behavior of $n$.

In order to verify if the same behavior at the singular point could be observed performing the integration in both directions, from $(0,1)$ to $(1,0)$ and from $(1,0)$ 

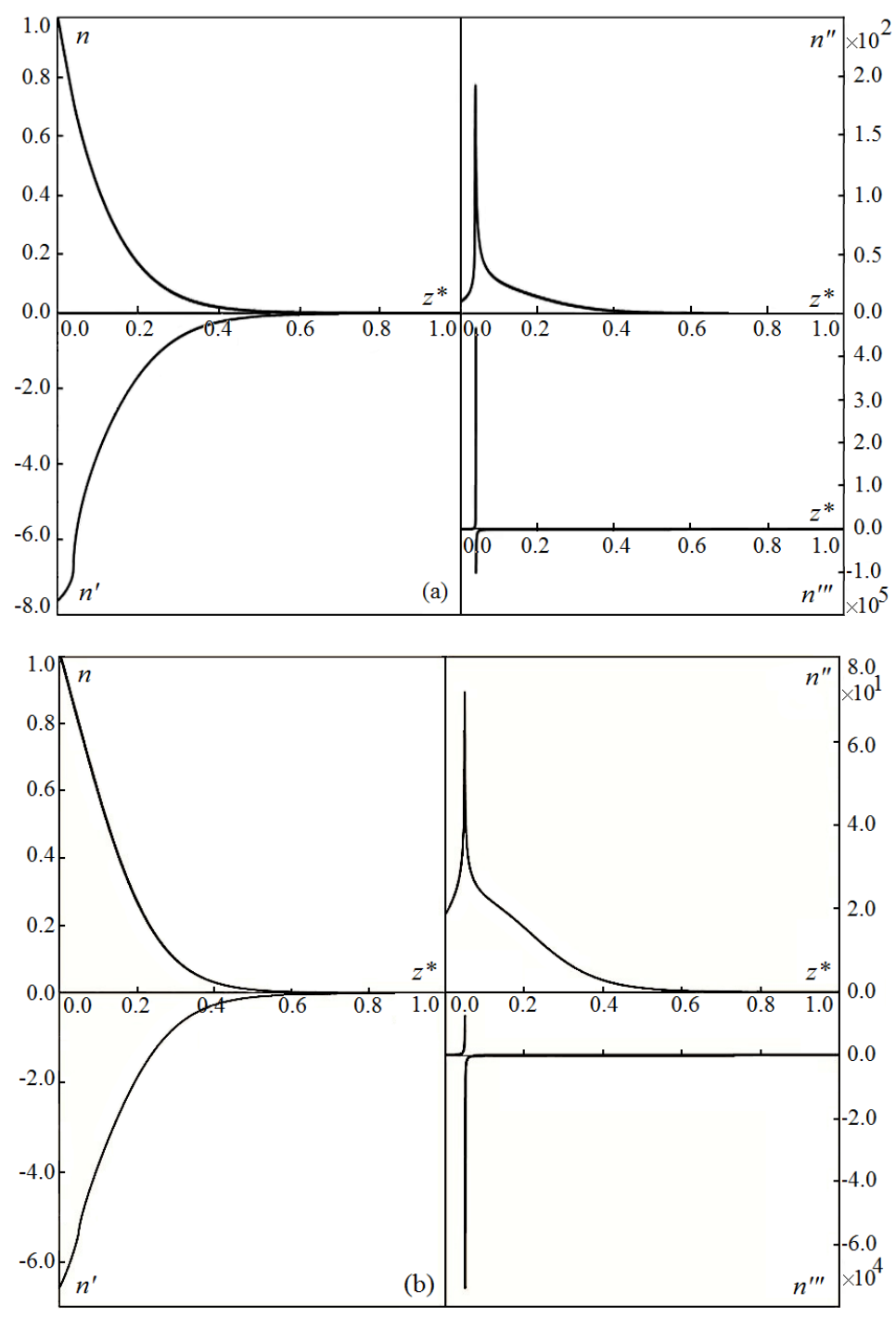

Figure 5: Smooth behavior of $n$, despite the behavior of the higher derivatives. (a) $k=0.003, A=0.5465, n^{\prime \prime \prime}(1) \approx 0.0338, n(0) \approx 1.0001$,

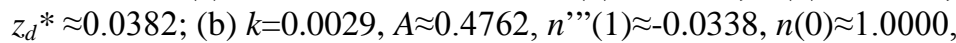
$z_{d}^{*} \approx 0.0506$, and in both cases $\Delta z^{*}=1 \times 10^{-3}$.

to $(0,1)$, detailed calculations were done refining the iteration steps closer to $z_{d}$. In the present test, having started with $\Delta z^{*}=2 \times 10^{-4}$, the calculations were refined using $\Delta z^{*}=4 \times 10^{-8}$. The obtained left and right values of $n$ and its derivatives in the vicinity of $z_{d}$ *are shown in table 2. Also shown are the values of $A, k, n(0)$, 
$z_{d}{ }^{*}$, and the starting value of $n$ "'(1). As can be seen, the third derivative tends to $\pm \infty$, but the second derivative assumes apparent finite values at both sides (using the present $\left.\Delta z^{*}\right)$. The function $n$ ” must of course be perpendicular to the abscissa $\left(z^{*}\right)$, but not necessarily tends to $\pm \infty$. The difference between the left and right values may be consequence of the different slopes of $n$ "' at both sides of $z_{d}$. More refined calculations may lead to a single value, or to $-\infty$, in which case the integral of the third derivative would not be an improper convergent integral. However, the integral of the second derivative seems to be convergent, because no major problems were observed for $n$ ', which attained the same value from both sides of $z_{d}{ }^{*}$, guarantying the continuity and smoothness of $n$.

Table 2: $\quad n$ and its derivatives for the test performed following the two directions: from liquid to gas and from gas to liquid.

\begin{tabular}{|c|c|c|c|}
\hline \multicolumn{4}{|c|}{$\begin{array}{c}\text { Test conditions: } k=0.003, \mathrm{~A} \approx 0.3982, n ’ ”(1) \sim-0.0371, n " '(0) \sim-938,36, n(0) \sim 1.0000 \text {, } \\
z_{d}{ }^{*} \sim 0.05589370 \text {, first } \Delta z^{*}=2 \times 10^{-4} \text {, second } \Delta z^{*=}=4 \times 10^{-8}\end{array}$} \\
\hline Left side & \multicolumn{2}{|c|}{$N$} & Right side \\
\hline$z^{*}=0.05589368$ & 0.674780213 & 0.674780198 & $z^{*}=0.05589372$ \\
\hline \multicolumn{4}{|c|}{ n' } \\
\hline$z^{*}=0.05589368$ & -3.957550837 & -3.95752447 & $z^{*}=0.05589372$ \\
\hline \multicolumn{4}{|c|}{$n "$} \\
\hline$Z^{*}=0.05589368$ & 3.884555433 & 0.70493587 & $z^{*}=0.05589372$ \\
\hline \multicolumn{4}{|c|}{$n "$} \\
\hline$z^{*}=0.05589368$ & -30201330.1 & 10879429.5 & $z^{*}=0.05589372$ \\
\hline
\end{tabular}

The integration used $\Delta z^{*}=2 \times 10^{-4}$ until the values of 0.0558 (from left, beginning at 0.0 ) and 0.0560 (from right, beginning at 1.0) were attained. Then $\Delta z^{*}=4 \times 10^{-8}$ was used until 0.05589368 (from left) and 0.05589372 (from right) were attained, generating table 2. Fig. 6 shows the scheme of this procedure.

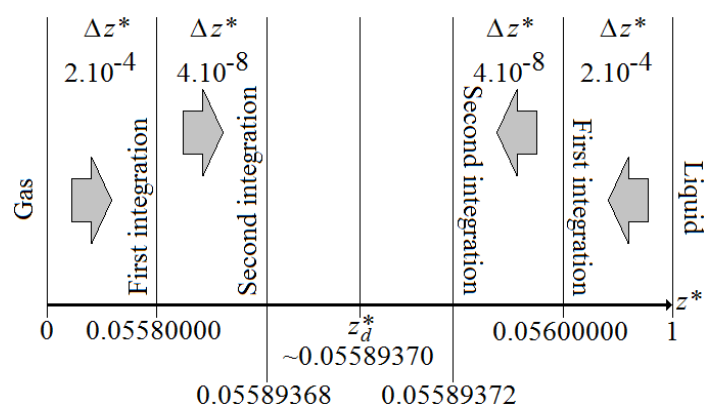

Figure 6: Schematic view of the refined steps around the singular point, $z_{d}$ *.

The results suggest that the general behavior of the $n$ profiles does not depend on the behavior of the higher derivatives, although these derivatives depend on the values of $k$ and $A$. Figs. 3 and 5, for $n$, show that variations are also observed in the profile of $n$, when $k$ and $A$ vary, but not affecting its general behavior (see fig. 7). 


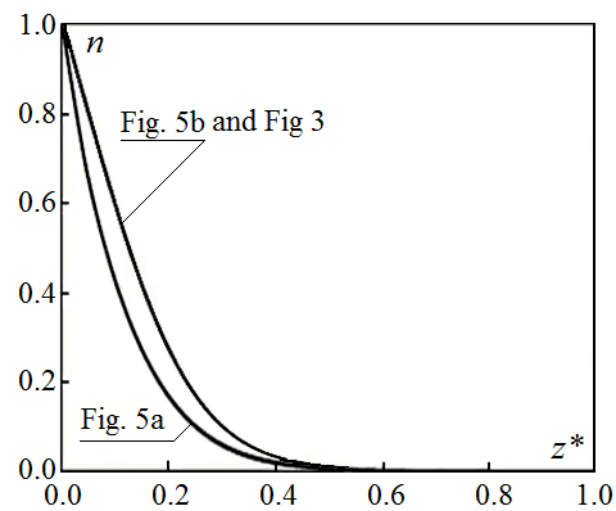

Figure 7: $\quad$ Comparison of $n$ profiles for different conditions given by $A$ and $k$.

\section{Conclusions}

The equations of one-dimensional mass transfer in turbulent flows expressed through the RSW approximation were tested for the case of a "constant reduction function". In this case, the set of equations may be reduced to a single nonlinear equation, which shows a singular point in the domain of calculus.

In order to perform predictions of the nondimensional mass concentration $(n)$ close to air-water interfaces, adequate boundary conditions were used, namely: $n(0)=1, n(1)=0, n^{\prime}(1)=0$. This conditions allowed proposing the new boundary condition $n$ "(1) $=k$, where $k$ is a nondimensional mass transfer coefficient.

The third derivative of the nondimensional concentration profile shows behaviors tending to $\pm \infty$ at the singular point (equal signs or opposite signs are possible). These behaviors affect the second derivative. But the first derivative of the nondimensional concentration shows a continuous profile, which leads to a continuous and smooth profile for the nondimensional concentration itself.

Different tests were performed in both directions: "from the liquid to the gas phase", and "from the gas to the liquid phase". For the present calculations, viable results for the nondimensional concentration profiles were obtained, independently of the behavior of the second and third derivatives, which, on their turn, depend on the parameters $A$ and $k$ existing in the formulation.

Comparisons with experimental data of the literature show that the calculated profiles agree with the observed results of the nondimensional concentration profile. Because viable results were obtained, it suggests that at least the integral of the second derivative is a case of a convergent improper integral.

\section{Acknowledgement}

The authors thank the National Council of Technological and Scientific Development - CNPq, Brazil, for financial support. 


\section{References}

[1] Davidson, P.A., Turbulence: an introduction for scientists and engineers. Oxford, University Press Inc., New York, pp. 3-27, 2004.

[2] Schulz, H.E.; Simões, A.L.A., and Janzen, J.G., Statistical approximations in gas-liquid mass transfer. In Komori, S; McGillis, W. and Kurose, R., Gas Transfer at Water Surfaces 2010, Kyoto University Press, Kyoto, Japan, pp. 208-221, 2011.

[3] Schulz, H.E.; Lopes Júnior, G.B.; Simões, A.L.A., and Lobosco, R.J. Onedimensional turbulent transfer using random square waves - scalar/velocity and velocity/velocity interactions (Chapter 1). Hydrodynamics- Advanced Topics, Intech open, Rijeka, Croatia, pp. 3-34, 2011.

[4] Janzen, J. G., Fluxo de massa na interface ar-água em tanques de grades oscilantes e detalhes de escoamentos turbulentos isotrópicos. (Gas transfer near the air-water interface in an oscillating-grid tanks and properties of isotropic turbulent flows - text in Portuguese). Doctoral thesis, 170 pp., University of São Paulo, Brazil, 2006.

[5] Schulz, H.E. and Janzen, J.G., Concentration fields near air-water interfaces during interfacial mass-transport: oxygen transport and random square wave analysis. Braz. J. Chem Eng. Vol. 26, n. 3, pp. 527-536, 2009.

[6] Komori, S., McGillis, W. and Kurose, R., Gas Transfer at Water Surfaces 2010, Kyoto University Press, Kyoto, Japan, 2011.

[7] Herlina, H. and Jirka, G. H., Experiments on gas transfer at the air-water interface induced by oscillating grid turbulence, J. Fluid Mech., 594, pp. 183-08, 2008.

[8] Janzen, J. G., Herlina, H., Jirka, G. H., Schulz, H. E. and Gulliver, J. S. Estimation of mass transfer velocity based on measured turbulence parameters. AIChE Journal, Vol. 56, n. 8, pp. 2005-2017, 2010.

[9] Janzen, J. G., Schulz, H. E. and Jirka, G., Turbulent gas flux measurements near the air-water interface in an oscillating-grid tank. In Komori, S; McGillis, W. and Kurose, R., Gas Transfer at Water Surfaces 2010, Kyoto University Press, Kyoto, Japan, pp. 65-77, 2011.

[10] Lopes Júnior, G. B., Organização de equações estatísticas para transferência de massa em processos turbulentos. (Organization of statistical equations for mass transfer in turbulent process - text in Portuguese). Dissertation, 109 pp., University of São Paulo, Brazil, 2012.

[11] Lopes Júnior, G.B. and Schulz, H.E., Análise de condições de contorno para a quantificação da transferência de massa unidimensional em regime turbulento (Analysis of boundary conditions for one-dimensional turbulent mass transfer - text in Portuguese). XXXIV National Congress of Applied and Computational Mathematics, Brazil, pp. 391-397, 2012. 